\title{
Concise syntheses of 5-substituted pyridazino[4,5-b]indolones and -diones
}

\author{
Norbert Haider* and Andrea Wobus \\ Department of Drug and Natural Product Synthesis, University of Vienna, Althanstraße 14, \\ A-1090 Vienna, Austria \\ E-mail: norbert.haider@univie.ac.at
}

Dedicated to Professor Guy Quéguiner on the occasion of his 70th birthday

\begin{abstract}
A series of 5-alkyl-4-methyl-2,5-dihydro-1H-pyridazino[4,5-b]indol-1-ones (3) was prepared from 2-acetylindole-2-carboxylic acid by a one-pot reaction ( $N$-alkylation, followed by ring closure with hydrazine). Similarly, various new 5-alkyl-2,3-dihydro-1H-pyridazino[4,5-b]indole1,4(5H)-diones (6) were obtained by alkylation and subsequent hydrazinolysis of dimethyl indole-2,3-dicarboxylate. A 5-alkyl representative (10) of the 3,5-dihydro-4H-pyridazino[4,5b]indol-4-one system was made available by two alternative pathways.
\end{abstract}

Keywords: Pyridazino[4,5-b]indoles, aza-carbolines, N-alkylation, hydrazinolysis

\section{Introduction}

The pyridazino[4,5-b]indole scaffold, due to its bio-isosterism with $\beta$-carboline as well as $\gamma$ carboline, has found considerable pharmaceutical interest as the core structure of a wide variety of bio-active compounds. ${ }^{1-9}$ During the past few years, we have investigated the synthesis and biological activity of various new representatives of this "aza-carboline" ring system, mainly focusing on potential antitumor agents. ${ }^{10-12}$ The title ring system now became interesting also in the context of an ongoing program in search of new and selective inhibitors of copper-containing amine oxidases. ${ }^{13}$ Based on preliminary structure-activity information, ${ }^{14}$ the need arose to prepare a focused compound library of indole-fused pyridazinones and pyridazinediones bearing various alkyl substituents at the indole nitrogen. Despite their simplicity, surprisingly few representatives of this general structure have been known so far. ${ }^{10,15-18}$ Here, we wish to report on short and convenient methods for the synthesis of such tricyclic compounds. 


\section{Results and Discussion}

5-Alkyl-4-methyl-2,5-dihydro-1H-pyridazino[4,5-b]indol-1-ones, representing one of the envisaged target structures, in principle can be prepared by selective monoalkylation of $\mathrm{N}-5$ in the parent compound with stoichiometric amounts of alkylating agents, as we had shown recently. ${ }^{10}$ However, yields are low and cannot be improved by employment of excess reagent, as this would result in the formation of 2,5-disubstituted products. ${ }^{10}$ Therefore, introduction of the desired alkyl residues at the indole nitrogen is preferentially accomplished before the pyridazinone unit is formed, using the precursor, 2-acetylindole-3-carboxylic $\operatorname{acid}^{19}(\mathbf{1})$ as the substrate (Scheme 1). Here, a larger excess of alkylating agent (generally an alkyl iodide, with the exception of benzyl bromide) can be safely employed, effecting N-alkylation and esterification of the carboxylic group at the same time. The resulting ester functionality, in turn, offers the additional advantage of facilitating the subsequent ring-closure reaction with hydrazine, because free carboxylic acids of this type have been known to undergo concurrent decarboxylation very easily. ${ }^{20}$ Although also this procedure gives only low to moderate yields, the sequence can be carried out very conveniently in a one-pot manner and opens a simple and short access to compounds of type 3 which are free of any contamination by 2,5-disubstituted derivatives.

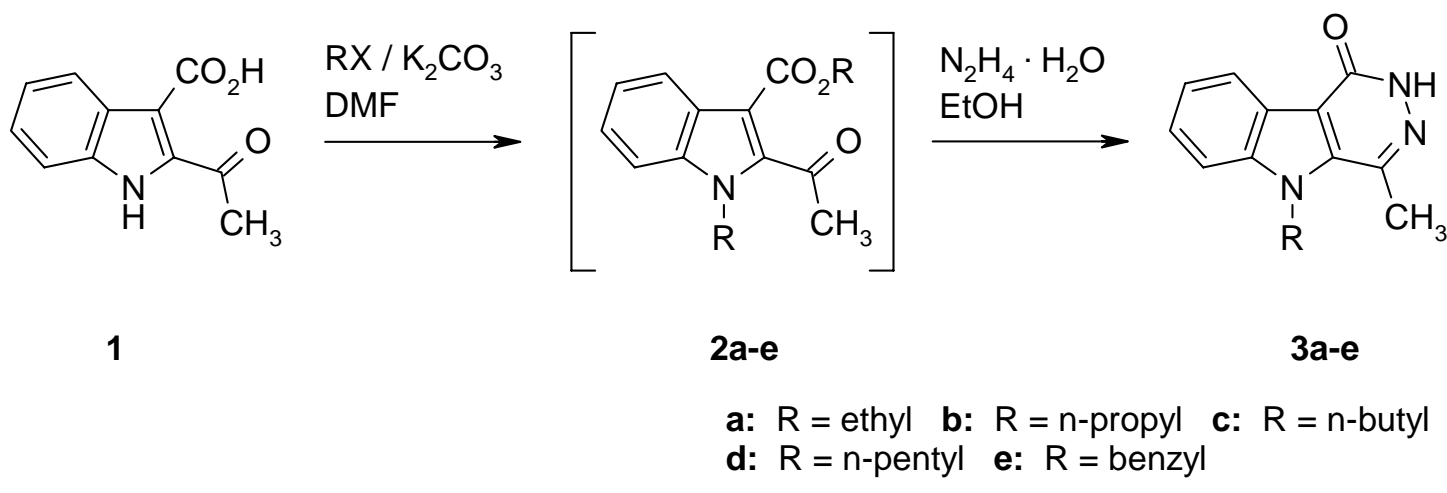

\section{Scheme 1}

For the preparation of a series of 5-alkyl-substituted 1,4-dihydroxypyridazino[4,5-b]indoles (or their oxo tautomers, respectively), an analogous strategy was chosen. Thus, dimethyl indole2,3-dicarboxylate ${ }^{21}$ was first $\mathrm{N}$-alkylated with an excess of the appropriate alkyl iodide (or benzyl bromide or allyl bromide, respectively) in the presence of potassium carbonate in dimethylformamide solution. Here, the intermediate $N$-alkyl esters $\mathbf{5}$ were extractively isolated prior to condensation with hydrazine hydrate which then gave the pyridazino-indole derivatives 6a-f in satisfactory yields. 


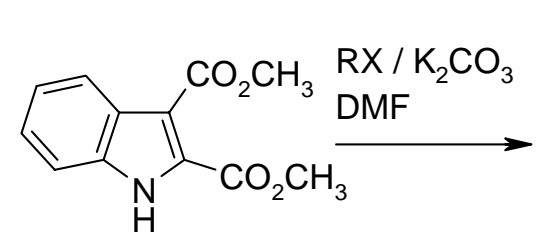

4

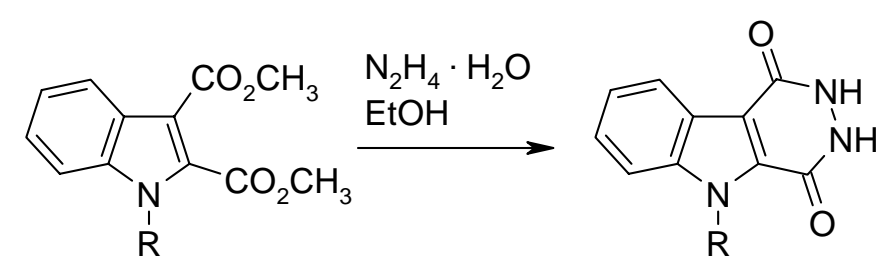

5a-f 6a-f

a: $R=$ ethyl b: $R=n$-propyl $\quad$ c: $R=n$-butyl

d: $R=$ n-pentyl e: $R=$ benzyl f: $R=$ allyl

\section{Scheme 2}

From the pyridazinediones (tautomeric dihydroxypyridazines or hydroxypyridazinones, respectively) of type 6, also mono-oxygenated compounds should be easily accessible by a sequence involving transformation of $\mathbf{6}$ into the corresponding dichloropyridazine, followed by monosubstitution with hydrazine, oxidative dehydrazination, and finally hydrolysis of the remaining chloro function. This approach was successfully exemplified, starting from the $\mathrm{N}$ propyl derivative $\mathbf{6 b}$, as shown in Scheme 3. Heating with phosphorus oxychloride smoothly afforded the dichloro compound 7 in $82 \%$ yield. Hydrazinolysis of 7 indeed gave a monohydrazino-monochloro product regioselectively. An analogous transformation of the 5unsubstituted dichloro congener, leading to a 1-chloro-4-hydrazino compound, had been previously reported by Monge and coworkers. ${ }^{22}$ Interestingly, in our case the regioselectivity of this substitution was found to be completely reversed, leading to the 4-chloro-1-hydrazino derivative 8 exclusively. Obviously, steric shielding of the 4-position by the adjacent $\mathrm{N}$-alkyl residue is responsible for the observed preferential attack of the nucleophile at $\mathrm{C}-1$ rather than at C-4 (as in Monge's 5-unsubstituted compound). The position of the newly introduced hydrazino group was firmly established after its replacement by hydrogen (compound 9) which is ideally suited for an NOE experiment, thus proving the close distance between H-9 and this pyridazine $\mathrm{H}$ atom. The transformation of $\mathbf{8}$ into $\mathbf{9}$ was accomplished by treatment with mercuric oxide in aqueous suspension in analogy to previous protocols. ${ }^{23,24}$ The final hydrolysis step, affording the new pyridazinone 10, succeeded by heating the chloropyridazine in acetic acid. ${ }^{25}$ As a more convenient synthesis of 10, which moreover avoids the use of toxic mercuric oxide, we elaborated a sequence starting from $N$-propylindole-2-carboxylic acid ethyl $\operatorname{ester}^{26}(\mathbf{1 1})$. This compound could be easily formylated at C-3 with Vilsmeier-Haack reagent under the conditions reported for similar substrates. ${ }^{27}$ As expected, the formyl ester $\mathbf{1 2}$ was found to cyclize smoothly with hydrazine hydrate in ethanolic solution to afford the tricycle $\mathbf{1 0}$ in good yield. 
<smiles>CCCn1c(C(=O)OCC)cc2ccccc21</smiles>

\section{Scheme 3}

In conclusion, a variety of new indole-fused pyridazinones and pyridazinediones bearing small to medium-sized alkyl residues at the indole nitrogen were made accessible by short and convenient synthetic pathways. Preliminary in-vitro tests for inhibition of certain amine oxidases showed only weak activity of the new compounds, further screenings for biological activity are in progress.

\section{Experimental Section}

General Procedures. Melting points were determined on a Kofler hot-stage microscope. IR spectra (KBr pellets) were recorded on a Perkin-Elmer 1605 FT-IR instrument. ${ }^{1} \mathrm{H}-\mathrm{NMR}$ and ${ }^{13} \mathrm{C}$-NMR spectra were recorded on a Varian Unityplus $300(300 \mathrm{MHz}, 75 \mathrm{MHz})$ and on a Bruker Avance DPX $200(200 \mathrm{MHz}, 50 \mathrm{MHz})$ spectrometer ( $\delta$ values in ppm). Mass spectra were obtained on a Shimadzu QP 5050A DI 50 instrument, high-resolution mass spectra (HRMS) were recorded on a Finnigan MAT 8230 spectrometer at the Institute of Organic Chemistry, University of Vienna. For column chromatography, Merck Kieselgel 60 (0.063-0.200 $\mathrm{mm}$ ) was used. Microanalyses were performed at the Microanalytical Laboratory, Faculty of Chemistry, University of Vienna.

5-Alkyl-4-methyl-2,5-dihydro-1H-pyridazino[4,5-b]indol-1-ones (3). General procedure. To a solution of 2-acetylindole-3-carboxylic $\operatorname{acid}^{19}(0.203 \mathrm{~g}, 1 \mathrm{mmol})$ in dry DMF $(10 \mathrm{~mL})$ was added $\mathrm{K}_{2} \mathrm{CO}_{3}(0.280 \mathrm{~g}, 2 \mathrm{mmol})$ and the appropriate alkyl iodide $(25-35 \mathrm{mmol}$; for $3 \mathbf{e}$, benzyl 
bromide was used), and the mixture was stirred at RT in a closed vessel for the time given below. The volatile components were removed under reduced pressure and the residue was taken up in a mixture of hydrazine monohydrate $(3 \mathrm{~mL}, 60 \mathrm{mmol})$ and $\mathrm{EtOH}(10 \mathrm{~mL})$. The solution was refluxed for $24 \mathrm{~h}$, then it was poured into ice-water $(100 \mathrm{~mL})$, acidified $(\mathrm{pH} 2-3)$ and kept in the refrigerator for at least $0.5 \mathrm{~h}$. The precipitate was collected by filtration, washed with water, and dried. Recrystallisation from EtOH gave the products as almost colorless crystals.

5-Ethyl-4-methyl-2,5-dihydro-1H-pyridazino[4,5-b]indol-1-one (3a). Reaction time: $200 \mathrm{~h}$, yield: $0.045 \mathrm{~g}(20 \%), \mathrm{mp} 285^{\circ} \mathrm{C}$; Anal. Calcd. $\mathrm{C}_{13} \mathrm{H}_{13} \mathrm{~N}_{3} \mathrm{O}$ : C, 68.70; H, 5.77; N, 18.49. Found: C, 68.53; H, 5.58; N, 18.63. IR (KBr, cm $\left.{ }^{-1}\right) 3222,2977,2924,1651,1456,1399,1212,752 ;{ }^{1} \mathrm{H}$ NMR (300 MHz, DMSO-d 6 ) $\delta 12.48(\mathrm{~s}, 1 \mathrm{H}, \mathrm{NH}), 8.25(\mathrm{~d}, J=8.2 \mathrm{~Hz}, 1 \mathrm{H}, 9-\mathrm{H}), 7.79(\mathrm{~d}, J=8.2$ $\mathrm{Hz}, 1 \mathrm{H}, 6-\mathrm{H}), 7.57-7.50(\mathrm{~m}, 1 \mathrm{H}, 7-\mathrm{H}), 7.38-7.32(\mathrm{~m}, 1 \mathrm{H}, 8-\mathrm{H}), 4.63(\mathrm{q}, J=7.2 \mathrm{~Hz}, 2 \mathrm{H}$, $\left.\mathrm{NCH}_{2} \mathrm{CH}_{3}\right), 2.75\left(\mathrm{~s}, 3 \mathrm{H}, 4-\mathrm{CH}_{3}\right), 1.38\left(\mathrm{t}, J=7.2 \mathrm{~Hz}, 3 \mathrm{H}, \mathrm{NCH}_{2} \underline{\mathrm{C}}_{3}\right) ;{ }^{13} \mathrm{C}$ NMR $(50 \mathrm{MHz}$, DMSO-d $\left._{6}\right) \delta 159.3,138.3,136.2,134.1,126.3,122.0,121.9,111.5,110.9,20.0,15.8 ; \mathrm{MS} \mathrm{m} / \mathrm{z}$ : 228 (10\%), $227\left(\mathrm{M}^{+}, 64\right), 212$ (48), 183 (10), 149 (8), 114 (18), 95 (16), 81 (54), 69 (100), 57 (29), 55 (31).

4-Methyl-5-propyl-2,5-dihydro-1H-pyridazino[4,5-b]indol-1-one (3b). Reaction time: $120 \mathrm{~h}$, yield: $0.108 \mathrm{~g}(45 \%), \mathrm{mp} 268^{\circ} \mathrm{C}$; Anal. Calcd. $\mathrm{C}_{14} \mathrm{H}_{15} \mathrm{~N}_{3} \mathrm{O}: \mathrm{C}, 69.69 ; \mathrm{H}, 6.27 ; \mathrm{N}, 17.41$. Found: C, 69.59; H, 6.22; N, 17.41. IR (KBr, cm $\left.{ }^{-1}\right) 3071,2922,1643,1456,1399,1206,1117,767 ;{ }^{1} \mathrm{H}$ NMR (300 MHz, DMSO-d 6 ) $\delta 12.49$ (s, 1H, NH), 8.25 (dd, $J=7.3 \mathrm{~Hz}, 0.5 \mathrm{~Hz}, 1 \mathrm{H}, 9-\mathrm{H}), 7.82$ $(\mathrm{d}, J=8.4 \mathrm{~Hz}, 1 \mathrm{H}, 6-\mathrm{H}), 7.56-7.50(\mathrm{~m}, 1 \mathrm{H}, 7-\mathrm{H}), 7.35(\mathrm{t}, J=7.4 \mathrm{~Hz}, 1 \mathrm{H}, 8-\mathrm{H}), 4.53(\mathrm{t}, J=7.6$ $\mathrm{Hz}, 2 \mathrm{H}, \mathrm{NC}_{2} \mathrm{CH}_{2} \mathrm{CH}_{3}$ ), 2.73 (s, 3H, 4- $\mathrm{CH}_{3}$ ), 1.80 (sextet, $J=7.4 \mathrm{~Hz}, 2 \mathrm{H}, \mathrm{NCH}_{2} \mathrm{C}_{2} \mathrm{CH}_{3}$ ), 0.93 $\left(\mathrm{t}, J=7.4 \mathrm{~Hz}, 3 \mathrm{H}, \mathrm{NCH}_{2} \mathrm{CH}_{2} \mathrm{C}_{3}\right) ;{ }^{13} \mathrm{C} \mathrm{NMR}\left(50 \mathrm{MHz}, \mathrm{DMSO}-\mathrm{d}_{6}\right) \delta 159.3,138.8,136.4,134.0$, 126.2, 121.9, 121.8, 121.7, 111.5, 111.2, 45.4, 24.0, 20.1, 10.9; MS m/z: $241\left(\mathrm{M}^{+}, 41 \%\right), 212$ (74), 149 (10), 115 (16), 95 (17), 81 (49), 69 (100), 57 (40), 55 (34).

5-Butyl-4-methyl-2,5-dihydro-1H-pyridazino[4,5-b]indol-1-one (3c). Reaction time: $120 \mathrm{~h}$, yield: $0.125 \mathrm{~g}(49 \%), \mathrm{mp} 245^{\circ} \mathrm{C}$; Anal. Calcd. $\mathrm{C}_{15} \mathrm{H}_{17} \mathrm{~N}_{3} \mathrm{O}: \mathrm{C}, 70.56 ; \mathrm{H}, 6.71 ; \mathrm{N}, 16.46$. Found: C, 70.31; H, 6.58; N, 16.37. IR (KBr, $\left.\mathrm{cm}^{-1}\right)$ 3146, 3070, 2956, 2920, 1642, 1524, 1456, 1399, 1193, 1118, 767; ${ }^{1} \mathrm{H}$ NMR (300 MHz, DMSO-d 6 ) $\delta 12.49$ (s, $\left.1 \mathrm{H}, \mathrm{NH}\right), 8.25(\mathrm{~d}, J=8.1 \mathrm{~Hz}, 1 \mathrm{H}$, 9-H), $7.78(\mathrm{~d}, J=8.1 \mathrm{~Hz}, 1 \mathrm{H}, 6-\mathrm{H}), 7.56-7.49(\mathrm{~m}, 1 \mathrm{H}, 7-\mathrm{H}), 7.35$ (t, $J=7.5 \mathrm{~Hz}, 1 \mathrm{H}, 8-\mathrm{H}), 4.55$ (t, $\left.J=7.8 \mathrm{~Hz}, 2 \mathrm{H}, \mathrm{NCH}_{2} \mathrm{CH}_{2} \mathrm{CH}_{2} \mathrm{CH}_{3}\right), 2.73\left(\mathrm{~s}, 3 \mathrm{H}, 4-\mathrm{CH}_{3}\right), 1.75$ (quintet, $J=7.8,2 \mathrm{H}$, $\mathrm{NCH}_{2} \mathrm{CH}_{2} \mathrm{CH}_{2} \mathrm{CH}_{3}$ ), 1.37 (sextet, $J=7.5 \mathrm{~Hz}, 2 \mathrm{H}, \mathrm{NCH}_{2} \mathrm{CH}_{2} \mathrm{C}_{2} \mathrm{CH}_{3}$ ), 0.91 (t, $J=7.5 \mathrm{~Hz}, 3 \mathrm{H}$, $\left.\mathrm{NCH}_{2} \mathrm{CH}_{2} \mathrm{CH}_{2} \mathrm{CH}_{3}\right) ;{ }^{13} \mathrm{C}$ NMR $(75 \mathrm{MHz}$, DMSO-d 6 ) $\delta 159.2,138.7,136.3,133.9,126.1,121.9$, 121.7, 111.5, 111.0, 43.9, 32.7, 20.0, 19.5, 13.5; MS m/z: 255 (M+, 21\%), 212 (42), 115 (8), 95 (6), 81 (21), 71 (14), 69 (38), 58 (100).

4-Methyl-5-pentyl-2,5-dihydro-1H-pyridazino[4,5-b]indol-1-one (3d). Reaction time: $120 \mathrm{~h}$, yield: $0.116 \mathrm{~g}(43 \%), \mathrm{mp} 229^{\circ} \mathrm{C}$; Anal. Calcd. $\mathrm{C}_{16} \mathrm{H}_{19} \mathrm{~N}_{3} \mathrm{O}$ : C, 71.35; H, 7.11; N, 15.60. Found: C, 71.46; H, 7.22; N, 15.60. IR (KBr, cm $\left.{ }^{-1}\right) 3069,2924,2863,1643,1456,1397,1172,768 ;{ }^{1} \mathrm{H}$ NMR (300 MHz, DMSO-d 6 ) $\delta 12.49(\mathrm{~s}, 1 \mathrm{H}, \mathrm{NH}), 8.25(\mathrm{~d}, J=8.2 \mathrm{~Hz}, 1 \mathrm{H}, 9-\mathrm{H}), 7.80(\mathrm{~d}, J=8.2$ $\mathrm{Hz}, 1 \mathrm{H}, 6-\mathrm{H}), 7.56-7.50(\mathrm{~m}, 1 \mathrm{H}, 7-\mathrm{H}), 7.35(\mathrm{t}, J=7.3 \mathrm{~Hz}, 1 \mathrm{H}, 8-\mathrm{H}), 4.56(\mathrm{t}, J=7.9 \mathrm{~Hz}, 2 \mathrm{H}$, $\mathrm{NC}_{2} \mathrm{CH}_{2} \mathrm{CH}_{2} \mathrm{CH}_{2} \mathrm{CH}_{3}$ ), 2.74 (s, $\left.3 \mathrm{H}, \quad 4-\mathrm{CH}_{3}\right), 1.77$ (quintet, $J=7.5 \mathrm{~Hz}, 2 \mathrm{H}$, 
$\left.\mathrm{NCH}_{2} \mathrm{C}_{2} \mathrm{CH}_{2} \mathrm{CH}_{2} \mathrm{CH}_{3}\right), 1.37-1.31$ (m, 4H, $\left.\mathrm{NCH}_{2} \mathrm{CH}_{2} \mathrm{C}_{2} \mathrm{C}_{2} \mathrm{CH}_{3}\right), 0.85$ (t, $J=7.1 \mathrm{~Hz}, 3 \mathrm{H}$, $\left.\mathrm{NCH}_{2} \mathrm{CH}_{2} \mathrm{CH}_{2} \mathrm{CH}_{2} \underline{\mathrm{C}}_{3}\right) ;{ }^{13} \mathrm{C}$ NMR (75 MHz, DMSO-d 6 ) $\delta 159.2,138.7,136.3,133.9,126.1$, 121.9, 121.7, 111.5, 111.0, 44.0, 30.3, 28.3, 21.7, 20.0, 13.8; MS m/z: $270(10 \%), 269\left(\mathrm{M}^{+}, 51\right)$, 241 (19), 213 (16), 212 (100), 183 (13), 155 (10), 115 (12), 71 (13), 57 (21), 55 (13).

5-Benzyl-4-methyl-2,5-dihydro-1H-pyridazino[4,5-b]indol-1-one (3e). Reaction time: $96 \mathrm{~h}$, yield: $0.058 \mathrm{~g}(20 \%)$, mp $243^{\circ} \mathrm{C}$; Anal. Calcd. $\mathrm{C}_{18} \mathrm{H}_{15} \mathrm{~N}_{3} \mathrm{O}: \mathrm{C}, 74.72 ; \mathrm{H}, 5.23 ; \mathrm{N}, 14.52$. Found: C, 74.47; H, 5.41; N, 14.37. IR (KBr, cm $\left.{ }^{-1}\right) 3160,2985,2916,1644,1452,1394,1169,753 ;{ }^{1} \mathrm{H}$ NMR (300 MHz, DMSO-d 6 ) $\delta 12.57$ (s, 1H, NH), 8.31 (dd, $J=7.8 \mathrm{~Hz}, 0.8 \mathrm{~Hz}, 1 \mathrm{H}, 9-\mathrm{H}), 7.74$ (dd, $J=7.8 \mathrm{~Hz}, 0.9 \mathrm{~Hz}, 1 \mathrm{H}, 6-\mathrm{H}), 7.53-7.47$ (m, 1H, 7-H), 7.38 (t, $J=7.0 \mathrm{~Hz}, 1 \mathrm{H}, 8-\mathrm{H}), 7.33-$ 7.21 (m, 3H, phenyl-H), 6.93 (d, J = 7.2 Hz, 2H, phenyl-H), 5.92 (s, 2H, $\mathrm{PhC}_{2}$ ), 2.54 (s, 3H, 4$\mathrm{CH}_{3}$ ); ${ }^{13} \mathrm{C}$ NMR (50 MHz, DMSO-d 6 ) $\delta 159.3,139.3,138.0,136.9,134.1,129.0,127.4,126.6$, 125.2, 122.2, 121.9, 121.8, 112.0, 111.1, 47.1, 19.6; MS m/z: 290 (13\%), 289 (M+ 57$), 255$ (3), 212 (6), 199 (9), 140 (5), 115 (12), 92 (24), 91 (100), 65 (23).

5-Alkyl-2,3-dihydro-1H-pyridazino[4,5-b]indole-1,4(5H)-diones (6). General procedure. To a solution of dimethyl indole-2,3-dicarboxylate ${ }^{21}(0.233 \mathrm{~g}, 1 \mathrm{mmol})$ in dry DMF $(10 \mathrm{~mL})$ was added $\mathrm{K}_{2} \mathrm{CO}_{3}(0.420 \mathrm{~g}, 3 \mathrm{mmol})$ and the appropriate alkyl iodide $(25-35 \mathrm{mmol}$; for 6e: benzyl bromide; for 6f: allyl bromide), and the mixture was stirred at RT in a closed vessel for $120 \mathrm{~h}$. The mixture was poured into water $(200 \mathrm{~mL})$ and it was extracted with $\mathrm{CH}_{2} \mathrm{Cl}_{2}(3 \times 50 \mathrm{~mL})$. The combined extracts were dried over $\mathrm{Na}_{2} \mathrm{SO}_{4}$ and evaporated, then the residue was taken up in a mixture of hydrazine monohydrate $(3 \mathrm{~mL}, 60 \mathrm{mmol})$ and $\mathrm{EtOH}(10 \mathrm{~mL})$. The solution was refluxed for $24 \mathrm{~h}$, then it was poured into ice-water $(100 \mathrm{~mL})$, acidified $(\mathrm{pH} 2-3)$ and kept in the refrigerator for at least $0.5 \mathrm{~h}$. The precipitate was collected by filtration, washed with water, and dried. Recrystallisation from EtOH gave the products as almost colorless crystals.

5-Ethyl-2,3-dihydro-1H-pyridazino[4,5-b]indole-1,4(5H)-dione (6a). Yield: $0.158 \mathrm{~g} \mathrm{(69 \% ),}$ $\mathrm{mp}>310^{\circ} \mathrm{C}$. Anal. Calcd. $\mathrm{C}_{12} \mathrm{H}_{11} \mathrm{~N}_{3} \mathrm{O}_{2}: \mathrm{C}, 62.87 ; \mathrm{H}, 4.84 ; \mathrm{N}, 18.33$. Found: C, 62.60; H, 4.61; N, 18.25. IR $\left(\mathrm{KBr}, \mathrm{cm}^{-1}\right) 2984,2880,1653,1617,1549,1335,1296,1108,738 ;{ }^{1} \mathrm{H}$ NMR (300 MHz, DMSO-d $\left.{ }_{6}\right) \delta 11.57-11.51$ (br s, 2H, NH), $8.13(\mathrm{~d}, J=8.1 \mathrm{~Hz}, 1 \mathrm{H}, 9-\mathrm{H}), 7.79(\mathrm{~d}, J=8.1$ $\mathrm{Hz}, 1 \mathrm{H}, 6-\mathrm{H}), 7.56-7.50(\mathrm{~m}, 1 \mathrm{H}, 7-\mathrm{H}), 7.37-7.31(\mathrm{~m}, 1 \mathrm{H}, 8-\mathrm{H}), 4.77$ (q, $J=6.9 \mathrm{~Hz}, 2 \mathrm{H}$, $\left.\mathrm{NCH}_{2} \mathrm{CH}_{3}\right), 1.35\left(\mathrm{t}, J=6.9 \mathrm{~Hz}, 3 \mathrm{H}, \mathrm{NCH}_{2} \mathrm{CH}_{3}\right) ;{ }^{13} \mathrm{C} \mathrm{NMR}\left(75 \mathrm{MHz}, \mathrm{DMSO}-\mathrm{d}_{6}\right) \delta 153.9,151.8$, 138.3, 130.2, 126.3, 122.1, 121.7, 120.9, 111.0, 110.9, 39.0, 15.8; MS m/z: 229 (M+ 7\%), 201 (7), 149 (6), 143 (7), 121 (6), 95 (13), 81 (46), 69 (100), 68 (17), 67 (17), 57 (29), 55 (34).

5-Propyl-2,3-dihydro-1H-pyridazino[4,5-b]indole-1,4(5H)-dione (6b). Yield: $0.170 \mathrm{~g}(70 \%)$, mp $292^{\circ}$ C. Anal. Calcd. $\mathrm{C}_{13} \mathrm{H}_{13} \mathrm{~N}_{3} \mathrm{O}_{2}$ : C, 64.19; H, 5.39; N, 17.27. Found: C, 64.29; H, 5.40; N, 17.28. IR (KBr, cm $\left.{ }^{-1}\right) 2963,1653,1551,1470,1292,1158,1113,860,737 ;{ }^{1} \mathrm{H}$ NMR (300 MHz, DMSO-d $\left._{6}\right) \delta 11.56-11.53($ br s, $2 \mathrm{H}, \mathrm{NH}), 8.13(\mathrm{~d}, J=8.2 \mathrm{~Hz}, 1 \mathrm{H}, 9-\mathrm{H}), 7.79(\mathrm{~d}, J=8.2 \mathrm{~Hz}, 1 \mathrm{H}$, 6-H), $7.52(\mathrm{t}, J=7.5 \mathrm{~Hz}, 1 \mathrm{H}, 7-\mathrm{H}), 7.34(\mathrm{t}, J=7.5 \mathrm{~Hz}, 1 \mathrm{H}, 8-\mathrm{H}), 4.71(\mathrm{t}, J=7.2 \mathrm{~Hz}, 2 \mathrm{H}$, $\mathrm{NC}_{2} \mathrm{CH}_{2} \mathrm{CH}_{3}$ ), 1.80 (sextet, $\left.J=7.2 \mathrm{~Hz}, 2 \mathrm{H}, \mathrm{NCH}_{2} \underline{\mathrm{C}}_{2} \mathrm{CH}_{3}\right), 0.82(\mathrm{t}, J=7.2 \mathrm{~Hz}, 3 \mathrm{H}$, $\mathrm{NCH}_{2} \mathrm{CH}_{2} \mathrm{C}_{3}$ ); ${ }^{13} \mathrm{C}$ NMR (50 MHz, DMSO-d ${ }_{6}$ ) $\delta 138.9,130.7,126.3,122.1,121.7,120.8$, 111.3, 45.3, 23.5, 10.8; MS m/z: 244 (6\%), $243\left(\mathrm{M}^{+}, 43\right), 214$ (36), 201 (100), 170 (16), 143 (54), 129 (13), 115 (25), 114 (21), 89 (18), 77 (17), 45 (26), 31 (35). 
5-Butyl-2,3-dihydro-1H-pyridazino[4,5-b]indole-1,4(5H)-dione (6c). Yield: $0.159 \mathrm{~g}(62 \%)$, mp $290^{\circ}$ C. Anal. Calcd. $\mathrm{C}_{14} \mathrm{H}_{15} \mathrm{~N}_{3} \mathrm{O}_{2}$ : C, 65.36; H, 5.88; N, 16.33. Found: C, 65.38; H, 5.79; N, 16.17. IR (KBr, cm $\left.{ }^{-1}\right) 2959,1653,1553,1472,1294,1113,735 ;{ }^{1} \mathrm{H}$ NMR (300 MHz, DMSO-d 6 ) $\delta 11.55$ (br s, 2H, NH), 8.13 (d, $J=8.2 \mathrm{~Hz}, 1 \mathrm{H}, 9-\mathrm{H}), 7.77$ (d, $J=8.2 \mathrm{~Hz}, 1 \mathrm{H}, 6-\mathrm{H}), 7.56-7.49$ $(\mathrm{m}, 1 \mathrm{H}, 7-\mathrm{H}), 7.34$ (t, $J=7.3 \mathrm{~Hz}, 1 \mathrm{H}, 8-\mathrm{H}), 4.77-4.71\left(\mathrm{~m}, 2 \mathrm{H}, \mathrm{NCH}_{2} \mathrm{CH}_{2} \mathrm{CH}_{2} \mathrm{CH}_{3}\right), 1.81-1.70$ (m, 2H, $\left.\mathrm{NCH}_{2} \mathrm{CH}_{2} \mathrm{CH}_{2} \mathrm{CH}_{3}\right), 1.32-1.19$ (m, 2H, $\left.\mathrm{NCH}_{2} \mathrm{CH}_{2} \mathrm{CH}_{2} \mathrm{CH}_{3}\right), 0.86$ (t, J = 7.2 Hz, 3H, $\left.\mathrm{NCH}_{2} \mathrm{CH}_{2} \mathrm{CH}_{2} \mathrm{CH}_{3}\right) ;{ }^{13} \mathrm{C} \mathrm{NMR}\left(75 \mathrm{MHz}, \mathrm{DMSO}-\mathrm{d}_{6}\right) \delta 153.8,151.7,138.8,130.6,126.2,122.1$, 121.6, 120.8, 111.2, 111.0, 43.7, 32.3, 19.3, 13.6; MS m/z: 258 (12\%), $257\left(\mathrm{M}^{+}, 69\right), 240$ (49), 228 (30), 215 (35), 214 (57), 201 (100), 170 (18), 143 (48), 115 (23), 89 (15).

5-Pentyl-2,3-dihydro-1H-pyridazino[4,5-b]indole-1,4(5H)-dione (6d). Yield: $0.192 \mathrm{~g}(71 \%)$, mp $309^{\circ}$ C. Anal. Calcd. $\mathrm{C}_{15} \mathrm{H}_{17} \mathrm{~N}_{3} \mathrm{O}_{2}$ : C, 66.40; H, 6.32; N, 15.49. Found: C, 66.51; H, 6.14; N, 15.22. IR $\left(\mathrm{KBr}, \mathrm{cm}^{-1}\right)$ 2956, 2930, 1653, 1550, 1472, 1294, 1156, 1113, 736; ${ }^{1} \mathrm{H}$ NMR $(300$ MHz, DMSO-d $)_{6} \delta 11.56-11.52$ (br s, 2H, NH), 8.13 (d, $\left.J=8.3 \mathrm{~Hz}, 1 \mathrm{H}, 9-\mathrm{H}\right), 7.77$ (d, $J=8.3$ $\mathrm{Hz}, 1 \mathrm{H}, 6-\mathrm{H}), 7.55-7.49(\mathrm{~m}, 1 \mathrm{H}, 7-\mathrm{H}), 7.34$ (t, $J=7.6 \mathrm{~Hz}, 1 \mathrm{H}, 8-\mathrm{H}), 4.73$ (t, $J=7.0 \mathrm{~Hz}, 2 \mathrm{H}$, $\left.\mathrm{NCH}_{2} \mathrm{CH}_{2} \mathrm{CH}_{2} \mathrm{CH}_{2} \mathrm{CH}_{3}\right), \quad 1.82-1.72\left(\mathrm{~m}, 2 \mathrm{H}, \quad \mathrm{NCH}_{2} \mathrm{CH}_{2} \mathrm{CH}_{2} \mathrm{CH}_{2} \mathrm{CH}_{3}\right), \quad 1.30-1.20$ (m, 4H, $\left.\mathrm{NCH}_{2} \mathrm{CH}_{2} \underline{\mathrm{C}}_{2} \mathrm{CH}_{2} \mathrm{CH}_{3}\right), 0.80\left(\mathrm{t}, J=7.0 \mathrm{~Hz}, 3 \mathrm{H}, \mathrm{NCH}_{2} \mathrm{CH}_{2} \mathrm{CH}_{2} \mathrm{CH}_{2} \mathrm{CH}_{3}\right) ;{ }^{13} \mathrm{C} \mathrm{NMR}(50 \mathrm{MHz}$, DMSO-d $\left._{6}\right) \delta 138.8,130.6,126.3,122.1,121.7,120.9,111.3,111.0,43.9,29.9,28.2,21.8$; MS m/z: 272 (8\%), $271\left(\mathrm{M}^{+}, 42\right), 254$ (48), 228 (24), 215 (30), 214 (53), 201 (100), 170 (19), 143 (43), 115 (23), 114 (23), 43 (33), 41 (40).

5-Benzyl-2,3-dihydro-1H-pyridazino[4,5-b]indole-1,4(5H)-dione (6e). Yield: $0.204 \mathrm{~g}$ (70\%), mp $315^{\circ}$ C. Anal. Calcd. $\mathrm{C}_{17} \mathrm{H}_{13} \mathrm{~N}_{3} \mathrm{O}_{2}$ : C, 70.09; H, 4.50; N, 14.42. Found: C, 69.92; H, 4.62; N, 14.23. IR ( $\left.\mathrm{KBr}, \mathrm{cm}^{-1}\right) 3028,2923,1653,1551,1471,1293,1153,1118,739 ;{ }^{1} \mathrm{H}$ NMR $(300$ MHz, DMSO-d $)_{6} \delta 11.68(\mathrm{~s}, 2 \mathrm{H}, \mathrm{NH}), 8.14(\mathrm{~d}, J=8.2 \mathrm{~Hz}, 1 \mathrm{H}, 9-\mathrm{H}), 7.70(\mathrm{~d}, J=8.2 \mathrm{~Hz}, 1 \mathrm{H}$, 6-H), 7.50-7.44 (m, 1H, 7-H), 7.36-7.30 (m, 1H, 8-H), 7.30-7.17 (m, 5H, phenyl-H), 6.00 (s, $\left.2 \mathrm{H}, \mathrm{PhCH}_{2}\right) ;{ }^{13} \mathrm{C} \mathrm{NMR}\left(50 \mathrm{MHz}, \mathrm{DMSO}-\mathrm{d}_{6}\right) \delta 138.8,137.8,130.6,128.6,127.4,126.9,126.6$, 122.2, 122.0, 121.0, 111.7, 111.2, 47.0; MS m/z: $291\left(\mathrm{M}^{+}, 12 \%\right), 214$ (4), 92 (12), 91 (95), 65 (17), 46 (22), 45 (57), 43 (17), 31 (100).

5-Allyl-2,3-dihydro-1H-pyridazino[4,5-b]indole-1,4(5H)-dione (6f). Yield: $0.205 \mathrm{~g}$ (85\%), mp $278^{\circ}$ C. Anal. Calcd. $\mathrm{C}_{13} \mathrm{H}_{11} \mathrm{~N}_{3} \mathrm{O}_{2}$ : C, 64.72; H, 4.60; N, 17.42: Found: C, 64.42; H, 4.80; N, 17.37. IR ( $\left.\mathrm{KBr}, \mathrm{cm}^{-1}\right) 2975,1653,1544,1472,1297,1149,1111,937,736 ;{ }^{1} \mathrm{H}$ NMR (300 MHz, DMSO-d $\left._{6}\right) \delta 11.60(\mathrm{~s}, 2 \mathrm{H}, \mathrm{NH}), 8.14(\mathrm{~d}, J=8.2 \mathrm{~Hz}, 1 \mathrm{H}, 9-\mathrm{H}), 7.70(\mathrm{~d}, J=8.2 \mathrm{~Hz}, 1 \mathrm{H}, 6-\mathrm{H})$, 7.54-7.48 (m, 1H, 7-H), 7.37-7.31 (m, 1H, 8-H), 6.10-5.96 (m, 1H, allyl 2'-H), 5.41 (d, J=5.4 Hz, 2H, allyl 1'-H), 5.10 (dd, $J=10.5 \mathrm{~Hz}, 1.5 \mathrm{~Hz}, 1 \mathrm{H}$, allyl 3'-H), 4.89 (dd, $J=17.4 \mathrm{~Hz}, 1.5 \mathrm{~Hz}$, $1 \mathrm{H}$, allyl 3'-H); ${ }^{13} \mathrm{C}$ NMR (50 MHz, DMSO-d 6 ) $\delta 138.8,134.0,130.5,126.4,122.1,121.9$, 120.9, 116.4, 111.5, 111.3, 46.1, 23.5, 10.8; MS m/z: 243 (35\%), $241\left(\mathrm{M}^{+}, 90\right), 224$ (59), 214 (36), 201 (100), 170 (23), 154 (36), 143 (62), 114 (46).

1,4-Dichloro-5-propyl-5H-pyridazino[4,5-b]indole (7). A mixture of compound $\mathbf{6 b}(0.486 \mathrm{~g}, 2$ $\mathrm{mmol})$ and $\mathrm{POCl}_{3}(12 \mathrm{~mL}, 128 \mathrm{mmol})$ was heated to $100^{\circ} \mathrm{C}$ for $4 \mathrm{~h}$. After cooling, the solution was slowly poured onto ice and it was basified with conc. ammonia. The precipitate was collected by filtration, washed with water, and dried to afford 7 as pale yellow crystals $(0.459 \mathrm{~g}$, 
82\%), mp $159^{\circ} \mathrm{C}$. Anal. Calcd. $\mathrm{C}_{13} \mathrm{H}_{11} \mathrm{Cl}_{2} \mathrm{~N}_{3} .0 .2 \mathrm{H}_{2} \mathrm{O}: \mathrm{C}, 55.03 ; \mathrm{H}, 4.05 ; \mathrm{N}, 14.81$. Found: $\mathrm{C}$, 55.01; H, 3.80; N, 14.69. IR (KBr, cm $\left.{ }^{-1}\right)$ 2960, 2873, 1619, 1553, 1493, 1425, 1331, 1237, 1079, $751 ;{ }^{1} \mathrm{H}$ NMR $\left(300 \mathrm{MHz}, \mathrm{DMSO}_{-} \mathrm{d}_{6}\right) \delta 8.52(\mathrm{~d}, J=8.2 \mathrm{~Hz}, 1 \mathrm{H}, 9-\mathrm{H}), 8.07(\mathrm{~d}, J=8.2 \mathrm{~Hz}, 1 \mathrm{H}$, 6-H), 7.88-7.82 (m, 1H, 7-H), $7.58(\mathrm{t}, J=7.7 \mathrm{~Hz}, 1 \mathrm{H}, 8-\mathrm{H}), 4.79(\mathrm{t}, J=7.5 \mathrm{~Hz}, 2 \mathrm{H}$, $\mathrm{NC}_{2} \mathrm{CH}_{2} \mathrm{CH}_{3}$ ), 1.87 (sextet, $J=7.5 \mathrm{~Hz}, 2 \mathrm{H}, \mathrm{NCH}_{2} \underline{\mathrm{C}}_{2} \mathrm{CH}_{3}$ ), $0.94(\mathrm{t}, J=7.5 \mathrm{~Hz}, 3 \mathrm{H}$, $\mathrm{NCH}_{2} \mathrm{CH}_{2} \mathrm{C}_{3}$ ); ${ }^{13} \mathrm{C}$ NMR (50 MHz, DMSO-d 6 ) $\delta$ 148.5, 141.0, 139.6, 133.1, 130.2, 123.0, 122.5, 118.6, 117.5, 112.0, 45.5, 23.8, 10.8; MS m/z: $283\left(\mathrm{M}^{+}, 2 \%\right), 281\left(\mathrm{M}^{+}, 22\right), 279\left(\mathrm{M}^{+}, 27\right)$, 252 (63), 250 (100), 180 (57), 138 (19), 99 (18), 63 (17).

4-Chloro-1-hydrazino-5-propyl-5H-pyridazino[4,5-b]indole (8). A mixture of compound 7 $(0.560 \mathrm{~g}, 2 \mathrm{mmol})$ and $100 \%$ hydrazine monohydrate $(20 \mathrm{~mL}, 400 \mathrm{mmol})$ was refluxed under an argon atmosphere for $6 \mathrm{~h}$. After cooling, the precipitate was collected by filtration, washed with water, and dried to afford $8(0.253 \mathrm{~g}, 46 \%)$ as pale yellow crystals, $\mathrm{mp} 159-160^{\circ} \mathrm{C}$. Anal. Calcd. $\mathrm{C}_{13} \mathrm{H}_{14} \mathrm{ClN}_{5}$ : C, 56.63; H, 5.12; N, 25.40. Found: C, 56.56; H, 5.11; N, 25.28. IR (KBr, $\left.\mathrm{cm}^{-1}\right)$ 3288, 2961, 2874, 1616, 1566, 1413, 1331, 1200, 1060, 1003, 751; ${ }^{1} \mathrm{H}$ NMR (300 MHz, DMSOd $\left.{ }_{6}\right) \delta 8.49(\mathrm{~d}, J=8.4 \mathrm{~Hz}, 1 \mathrm{H}, 9-\mathrm{H}), 8.05(\mathrm{~d}, J=8.4 \mathrm{~Hz}, 1 \mathrm{H}, 6-\mathrm{H}), 7.86-7.80(\mathrm{~m}, 1 \mathrm{H}, 7-\mathrm{H}), 7.57$ (t, $J=7.7 \mathrm{~Hz}, 1 \mathrm{H}, 8-\mathrm{H}$ ), 4.77 (t, $J=7.5 \mathrm{~Hz}, 2 \mathrm{H}, \mathrm{NC}_{2} \mathrm{CH}_{2} \mathrm{CH}_{3}$ ), 3.32 (br, $3 \mathrm{H}, \mathrm{NH}$ ), 1.86 (sextet, $\left.J=7.5 \mathrm{~Hz}, 2 \mathrm{H}, \mathrm{NCH}_{2} \mathrm{CH}_{2} \mathrm{CH}_{3}\right), 0.93\left(\mathrm{t}, J=7.5 \mathrm{~Hz}, 3 \mathrm{H}, \mathrm{NCH}_{2} \mathrm{CH}_{2} \mathrm{CH}_{3}\right) ;{ }^{13} \mathrm{C} \mathrm{NMR}(50 \mathrm{MHz}$, DMSO-d $\left._{6}\right) \delta 148.5,141.0,139.6,133.1,130.2,123.0,122.5,118.6,117.5,112.0,45.5,23.7$, 10.7; MS m/z: $277\left(\mathrm{M}^{+}, 16 \%\right), 275\left(\mathrm{M}^{+}, 49\right), 245$ (43), 216 (100), 180 (50), 168 (56), 153 (48), 126 (31), 114 (31), 77 (88), 51 (47).

4-Chloro-5-propyl-5H-pyridazino[4,5-b]indole (9). To a stirred suspension of yellow $\mathrm{HgO}$ $(0.432 \mathrm{~g}, 2 \mathrm{mmol})$ in water $(10 \mathrm{~mL})$ was added compound $8(0.275 \mathrm{~g}, 1 \mathrm{mmol})$ in small portions at RT. Stirring was continued for $2 \mathrm{~h}$, then the mixture was extracted several times with AcOEt. The combined extracts were dried over $\mathrm{Na}_{2} \mathrm{SO}_{4}$ and evaporated under reduced pressure. The dark oily residue was subjected to column chromatography (AcOEt) to afford $9(0.073 \mathrm{~g}, 30 \%)$ as a yellow-orange solid, $\mathrm{mp} 125-126^{\circ} \mathrm{C}$, which was used for the following step without further purification. ${ }^{1} \mathrm{H}$ NMR $\left(300 \mathrm{MHz}, \mathrm{DMSO}_{-} \mathrm{d}_{6}\right) \delta 9.97(\mathrm{~s}, 1 \mathrm{H}, 1-\mathrm{H}$, shows positive NOE on irradiation at $8.43 \mathrm{ppm}), 8.43(\mathrm{~d}, J=8.2 \mathrm{~Hz}, 1 \mathrm{H}, 9-\mathrm{H}), 7.96(\mathrm{~d}, J=8.2 \mathrm{~Hz}, 1 \mathrm{H}, 6-\mathrm{H}$, shows positive NOE on irradiation at $4.75 \mathrm{ppm}), 7.78-7.72(\mathrm{~m}, 1 \mathrm{H}, 7-\mathrm{H}), 7.49(\mathrm{t}, J=7.5 \mathrm{~Hz}, 1 \mathrm{H}, 8-\mathrm{H})$, $4.75\left(\mathrm{t}, J=7.5 \mathrm{~Hz}, 2 \mathrm{H}, \mathrm{NCH}_{2} \mathrm{CH}_{2} \mathrm{CH}_{3}\right.$ ), 1.86 (sextet, $J=7.5 \mathrm{~Hz}, 2 \mathrm{H}, \mathrm{NCH}_{2} \mathrm{CH}_{2} \mathrm{CH}_{3}$ ), 0.92 (t, $J=$ $\left.7.5 \mathrm{~Hz}, 3 \mathrm{H}, \mathrm{NCH}_{2} \mathrm{CH}_{2} \mathrm{CH}_{3}\right) ;{ }^{13} \mathrm{C} \mathrm{NMR}\left(50 \mathrm{MHz}, \mathrm{DMSO}-\mathrm{d}_{6}\right) \delta 144.2,140.9,139.6,132.0,129.7$, 122.2, 121.4, 118.5, 111.6, 45.4, 23.8, 10.8; MS m/z: $247\left(\mathrm{M}^{+}, 15 \%\right), 245\left(\mathrm{M}^{+}, 44 \%\right), 218(30)$, 216 (100), 180 (41), 153 (26), 126 (22), 75 (20), 63 (21), 51 (15).

Ethyl 1-propyl-1H-indole-2-carboxylate ${ }^{26}$ (11). A mixture of ethyl indole-2-carboxylate $(0.189$ g, $1 \mathrm{mmol}), \mathrm{K}_{2} \mathrm{CO}_{3}(0.420 \mathrm{~g}, 3 \mathrm{mmol})$, and propyl iodide $(3.0 \mathrm{~mL}, 31 \mathrm{mmol})$ in DMF $(10 \mathrm{~mL})$ was stirred at RT for $120 \mathrm{~h}$, then it was poured into water $(100 \mathrm{~mL})$ and extracted repeatedly with $\mathrm{Et}_{2} \mathrm{O}$. The combined extracts were washed with water, dried over $\mathrm{Na}_{2} \mathrm{SO}_{4}$, and evaporated to give $11(0.162 \mathrm{~g}, 70 \%)$ as a yellow oil. ${ }^{1} \mathrm{H}$ NMR $\left(300 \mathrm{MHz}, \mathrm{DMSO}-\mathrm{d}_{6}\right) \delta 7.66(\mathrm{~d}, J=8.2 \mathrm{~Hz}$, $1 \mathrm{H}, 4-\mathrm{H}), 7.57$ (dd, $J=8.2 \mathrm{~Hz}, 0.8 \mathrm{~Hz}, 1 \mathrm{H}, 7-\mathrm{H}), 7.34-7.28$ (m, 1H, 6-H), 7.26 (s, 1H, 3-H), 7.13-7.07 (m, 1H, 5-H), $4.50\left(\mathrm{t}, J=7.4 \mathrm{~Hz}, 2 \mathrm{H}, \mathrm{NC}_{2} \mathrm{CH}_{2} \mathrm{CH}_{3}\right), 4.30(\mathrm{q}, J=7.2 \mathrm{~Hz}, 2 \mathrm{H}$, 
$\mathrm{OC}_{2} \mathrm{CH}_{3}$ ), 1.69 (sextet, $J=7.4 \mathrm{~Hz}, 2 \mathrm{H}, \mathrm{NCH}_{2} \mathrm{CH}_{2} \mathrm{CH}_{3}$ ), 1.32 (t, $J=7.2 \mathrm{~Hz}, 3 \mathrm{H}, \mathrm{OCH}_{2} \underline{\mathrm{C}}_{3}$ ), $0.81\left(\mathrm{t}, J=7.4 \mathrm{~Hz}, 3 \mathrm{H}, \mathrm{NCH}_{2} \mathrm{CH}_{2} \mathrm{CH}_{3}\right) ;{ }^{13} \mathrm{C} \mathrm{NMR}\left(50 \mathrm{MHz}, \mathrm{DMSO}-\mathrm{d}_{6}\right) \delta 161.1,138.7,127.0$, $125.3,124.7,122.2,120.3,111.9,110.0,60.2,45.3,23.4,14.0,10.2 ; \mathrm{MS} \mathrm{m} / \mathrm{z}: 232(8 \%), 231$ $\left(\mathrm{M}^{+}, 48\right), 202$ (68), 174 (100), 143 (25), 130 (22), 116 (24), 115 (30), 89 (40), 77 (14), 63 (14); HRMS Calcd. $\mathrm{C}_{14} \mathrm{H}_{17} \mathrm{NO}_{2}: 231.1259$. Found: 231.1256.

Ethyl 3-formyl-1-propyl-1H-indole-2-carboxylate (12). To a stirred solution of $\mathrm{POCl}_{3}(0.3$ $\mathrm{mL}, 3.3 \mathrm{mmol})$ in dry DMF $(4 \mathrm{~mL})$ was added dropwise a solution of compound $11(0.231 \mathrm{~g}, 1$ mmol) in dry DMF $(2 \mathrm{~mL})$, then the mixture was heated to $110^{\circ} \mathrm{C}$ for $1 \mathrm{~h}$. It was then poured into water $(100 \mathrm{~mL})$, made alkaline with $\mathrm{Na}_{2} \mathrm{CO}_{3}$, and extracted with $\mathrm{Et}_{2} \mathrm{O}$. The combined extracts were washed with water, dried over $\mathrm{Na}_{2} \mathrm{SO}_{4}$, and evaporated to afford $12(0.218 \mathrm{~g}, 84 \%)$ as a yellow oil. ${ }^{1} \mathrm{H}$ NMR (300 MHz, DMSO-d 6 ) $\delta 10.42(\mathrm{~s}, 1 \mathrm{H}$, formyl-H), $8.30(\mathrm{~d}, J=8.0 \mathrm{~Hz}, 1 \mathrm{H}$, 4-H), 7.74 (d, $J=8.0 \mathrm{~Hz}, 1 \mathrm{H}, 7-\mathrm{H}), 7.47-7.40$ (m, 1H, 6-H), 7.36-7.30 (m, 1H, 5-H), 4.51-4.42 (m, $4 \mathrm{H}, \mathrm{NC}_{2} \mathrm{CH}_{2} \mathrm{CH}_{3}, \mathrm{OC}_{2} \mathrm{CH}_{3}$ ), 1.76 (sextet, $J=7.4 \mathrm{~Hz}, 2 \mathrm{H}, \mathrm{NCH}_{2} \mathrm{CH}_{2} \mathrm{CH}_{3}$ ), 1.38 (t, $J=7.1$ $\left.\mathrm{Hz}, 3 \mathrm{H}, \mathrm{OCH}_{2} \mathrm{CH}_{3}\right), 0.86\left(\mathrm{t}, J=7.4 \mathrm{~Hz}, 3 \mathrm{H}, \mathrm{NCH}_{2} \mathrm{CH}_{2} \mathrm{CH}_{3}\right) .{ }^{13} \mathrm{C} \mathrm{NMR}\left(50 \mathrm{MHz}, \mathrm{DMSO}-\mathrm{d}_{6}\right) \delta$ 187.4, 160.2, 137.1, 134.0, 125.8, 123.7, 123.7, 122.3, 118.5, 111.7, 62.1, 46.3, 23.2, 13.8, 10.8; MS m/z: 259 (M+, 18\%), 230 (100), 188 (23), 172 (26), 170 (67), 143 (13), 116 (31), 115 (31), 114 (27), 89 (28), 77 (13), 63 (12); HRMS Calcd. $\mathrm{C}_{15} \mathrm{H}_{17} \mathrm{NO}_{3}$ : 259.1208. Found: 259.1214.

\section{5-Propyl-3,5-dihydro-4H-pyridazino[4,5-b]indol-4-one (10).}

Method A. A solution of the chloro compound 9 (0.245 g, $1 \mathrm{mmol})$ in AcOH $(16 \mathrm{~mL})$ was refluxed for $8 \mathrm{~h}$, then it was evaporated under reduced pressure. The residue was triturated with water, filtered off and dried to give $10(0.068 \mathrm{~g}, 30 \%)$ as pale yellow crystals.

Method B. A solution of compound $12(0.227 \mathrm{~g}, 1 \mathrm{mmol})$ and 100\% hydrazine monohydrate (3 $\mathrm{mL}, 60 \mathrm{mmol})$ in EtOH $(3 \mathrm{~mL})$ was refluxed for $3 \mathrm{~h}$. The volatile components were removed under reduced pressure and the residue was taken up in water $(20 \mathrm{~mL})$, acidified $(\mathrm{pH} \mathrm{2-3})$ and kept in the refrigerator for $0.5 \mathrm{~h}$. Then the precipitate was collected by filtration, washed with water and dried to give $10(0.175 \mathrm{~g}, 77 \%)$ as almost colorless crystals, mp $207^{\circ} \mathrm{C}$. Anal. Calcd. $\mathrm{C}_{13} \mathrm{H}_{13} \mathrm{~N}_{3} \mathrm{O}: \mathrm{C}, 68.71 ; \mathrm{H}, 5.77 ; \mathrm{N}, 18.49$. Found: C, 68.48; H, 5.83; N, 18.49. IR (KBr, cm $\left.{ }^{-1}\right)$ 3166, 2960, 1649, 1520, 1462, 1343, 959, 736; ${ }^{1} \mathrm{H}$ NMR (300 MHz, DMSO-d 6 ) $\delta 12.9-12.5$ (br s, 1H, NH), $8.74(\mathrm{~s}, 1 \mathrm{H}, 1-\mathrm{H}), 8.18(\mathrm{~d}, J=8.2 \mathrm{~Hz}, 1 \mathrm{H}, 9-\mathrm{H}), 7.78$ (d, J=8.2 Hz, 1H, 6-H), 7.58$7.52(\mathrm{~m}, 1 \mathrm{H}, 7-\mathrm{H}), 7.37-7.31(\mathrm{~m}, 1 \mathrm{H}, 8-\mathrm{H}), 4.74\left(\mathrm{t}, J=7.2 \mathrm{~Hz}, 2 \mathrm{H}, \mathrm{NCH}_{2} \mathrm{CH}_{2} \mathrm{CH}_{3}\right.$ ), 1.80 (sextet, $\left.J=7.5 \mathrm{~Hz}, 2 \mathrm{H}, \mathrm{NCH}_{2} \mathrm{CH}_{2} \mathrm{CH}_{3}\right), 0.80\left(\mathrm{t}, J=7.2 \mathrm{~Hz}, 3 \mathrm{H}, \mathrm{NCH}_{2} \mathrm{CH}_{2} \mathrm{CH}_{3}\right) ;{ }^{13} \mathrm{C} \mathrm{NMR}(75 \mathrm{MHz}$, DMSO-d $\left._{6}\right) \delta 155.9,139.2,133.1,130.0,127.0,121.5,121.4,120.0,117.3,111.4,45.5,23.5$, 10.7; MS m/z: 227 (M+, 32\%), 198 (43), 185 (100), 129 (29), 128 (21), 115 (25), 101 (34), 75 (25).

\section{References and Footnotes}

1. Monge, A.; Aldana I.; Alvarez, T.; Losa, M. J.; Font, M.; Cenarruzabeitia, E.; Lasheras, B.; Frechilla, D.; Castiella, E.; Fernandez-Alvarez, E. Eur. J. Med. Chem. 1991, 26, 655. 
2. Lerch, U.; Kaiser, J. DE 3121137, 1982; Chem. Abstr. 1983, 98, 126140.

3. Monge, A.; Aldana, I.; Losa, M. J.; Font, M.; Cenarruzabeitia, E.; Castiella, E.; Frechilla, D.; Santiago, E.; Martínez de Irujo, J. J.; Alberdi, E.; López-Unzu, M. J. Arzneim.-Forsch. 1993, 43, 1175.

4. Monge, A.; Aldana, I.; Erro, A.; Parrado, P.; Font, M.; Alvarez, T.; Rocha, E.; FernandezAlvarez, E. An. R. Acad. Farm. 1985, 51, 485; Chem. Abstr. 1987, 107, 254.

5. Monge Vega, A.; Palop, J. A.; Martinez, M. T.; Fernandez-Alvarez, E. An. Quim. 1979, 75, 889; Chem. Abstr. 1980, 93, 2873.

6. Nantka-Namirski, P.; Ozdowska, Z. Acta Pol. Pharm. 1972, 29, 7; Chem. Abstr. 1972, 77, 101504.

7. Nantka-Namirski, P.; Ozdowska, Z. Acta Pol. Pharm. 1972, 29, 13; Chem. Abstr. 1972, 77, 101501.

8. Evanno, Y.; Dubois, L.; Sevrin, M.; Marguet, F.; Froissant, J.; Bartsch, R.; Gille, C. WO 9906406, 1999; Chem. Abstr. 1999, 130, 168385.

9. Font, M.; Monge, A.; Cuartero, A.; Elorriaga, A.; Martínez-Irujo, J. J.; Alberdi, E.; Santiago, E.; Prieto, I.; Lasarte, J. J.; Sarobe, P.; Borrás, F. Eur. J. Med. Chem. 1995, 30, 963.

10. El-Kashef, H.; Farghaly, A. A. H.; Floriani, S.; Haider, N. Arkivoc 2003, 198.

11. El-Kashef, H.; Farghaly, A. A. H.; Haider, N.; Wobus, A. Molecules 2004, 9, 849.

12. Haider, N.; Wobus, A. Heterocycles 2006, 68, 2549.

13. For a review on SSAO, an interesting representative of this enzyme family, see Mátyus, P.; Dajka-Halasz, B.; Földi, A.; Haider, N.; Barlocco, D.; Magyar, K. Curr. Med. Chem. 2004, $11,1285$.

14. Farghaly, A. A. H. Ph.D. thesis, Assiut University, 2001.

15. Güven A.; Jones, R. A. J. Chem. Res. (M) 1993, 2410.

16. Güven, A.; Jones, R. A. Tetrahedron 1993, 49, 11145.

17. Matsuo, I.; Takakuwa, T.; Kishii, K.; Harada, M.; Mitani, M. Jpn. Kokai Tokkyo Koho JP 08113574, 1996; Chem. Abstr. 1996, 125, 114662.

18. Monge, A.; Palop, J. A.; Goni, T.; Fernandez-Alvarez, E. J. Heterocyclic Chem. 1986, 23, 141.

19. Gorgos, V. I.; Zorin, L. M.; Zhungietu, G. I.; Rekhter, M. A. Chem. Heterocycl. Compd. (Engl. Transl.) 1983, 19, 1179.

20. Black, D. St. C.; Wong, L. C. H. J. Chem. Soc., Chem. Comm. 1980, 200.

21. Diels O.; Reese, J. Liebigs Ann. Chem. 1934, 511, 168.

22. Monge-Vega, A.; Aldana, I.; Ferandez-Alvarez, E. J. Heterocyclic Chem. 1981, 18, 1533.

23. Paul, D. B.; Rodda, H. J. Aust. J. Chem. 1968, 21, 1291.

24. Haider, N.; Käferböck, J. Heterocycles 2000, 53, 2527.

25. Dal Piaz, V.; Giovannoni, M. P.; Ciciani, G. Tetrahedron Lett. 1993, 34, 3903.

26. Hlasta, D. J., Luttinger, D., Perrone, M. H., Silbernagel, M. J., Ward, S. J., Haubrich, D. R. J. Med. Chem. 1987, 30, 1555.

27. Monge Vega, A.; Aldana, I.; Fernandez-Alvarez, E. Eur. J. Med. Chem. 1978, 13, 573. 